\title{
Analisador de fibras nervosas: um estudo sobre os resulta- dos falso-positivos
}

\author{
Nerve fiber analyzer:a study on the false-positive results
}

Jair Giampani Junior (1)

Bruno Campelo Leal (2)

Remo Susanna Junior ${ }^{(3)}$

\section{RESUMO}

Objetivos: Determinar a especificidade do analisador de fibras nervosasGDx em um grupo de pacientes normais, assim como os índices que mais comumente mostram-se alterados em pacientes deste grupo. Métodos: Foram submetidos a exame oftalmológico completo 50 pacientes normais (não-glaucomatosos) e na seqüência obtida a análise da camada de fibras nervosas destes pacientes, utilizando-se o software GDx (LDT). Foram excluídos os olhos que não perfaziam os critérios de inclusão e utilizado apenas um olho decada paciente após randomização, perfazendo um total de 34 olhos. Resultados e Conclusões: Obtivemos 20 exames normais e 14 exames alterados. $O$ índice com maior freqüência de alteração foi a simetria $(85,71 \%)$, e os com menor freqüência "inferior ratio" e "superior ratio" (0\% cada). A especificidade obtida foi de 58,8\%, elevando-se para 70,5\% após correção e análise dos dados. Devemos levantar a hipótese de um exame-falso positivo quando um paciente com exame ocular normal mostrar a simetria como único índice alterado ao GDx, elembrar do diagnóstico de glaucoma quando um paciente suspeito apresentar alteração de"inferior ratio".

Descritores: Fibras nervosas; Especificidade; Técnicas de diagnóstico oftalmológico; Lasers/uso diagnóstico.

\section{INTRODUÇÃO E OBJETIVOS}

A obtenção da imagem da camada de fibras nervosas (CFN) é conseguida no analisador de fibras nervosas GDx através da birrefringência que os axônios das células ganglionares apresentam à luz polarizada ${ }^{1}$. Esta birrefringência se deve à disposição paralela dos microtúbulos nos axônios daquelas células. A luz polarizada passa através da CFN retiniana, sofrendo um retardo que é proporcional à espessura da CFN.

Esta correlação foi demonstrada através de estudos anatomopatológicos em macacos que tiveram suas córneas e cristalinos removidos ${ }^{2}, \mathrm{e}$ em olhos humanos intactos ${ }^{1,3}$.

Estas estruturas podem porém, in vivo, polarizar a luz, afetando os resultados.

Com o objetivo de minimizar estes efeitos, foi incorporada ao aparelho a luz com espectro infravermelho $(780 \mathrm{~nm})$, e um dispositivo que compensa a polarização causada por aquelas estruturas birrefringentes.

Vários estudos mostram ser a análise da CFN pela polarimetria a laser um método promissor e com aplicabilidade clínica ${ }^{4-6}$.

Neste estudo, objetivamos determinar a especificidade do analisador de fibras nervosas em um grupo de pacientes não glaucomatosos (denominados normais), assim como os índices que mais freqüentemente são causa 
de exames alterados (denominados falsos-positivos) em pacientes pertencentes a este grupo.

\section{MÉTODOS}

Foram selecionados 94 olhos de 50 pacientes não glaucomatosos de diferentes faixas etárias, com idade variando de 18 a 71 anos, de ambos os sexos.

Os critérios de inclusão deste estudo foram pressão intraocular menor que $21 \mathrm{~mm} \mathrm{Hg}$, idade acima de 18 anos, ausência de história familiar de glaucoma, biomicroscopia sem sinais de pseudoesfoliação, dispersão pigmentar, câmara anterior rasa ou uveíte, AV corrigida igual ou melhor que 20/40 em ambos os olhos, erro refracional $\pm 5,0 \mathrm{DE}$ e uma escavação menor ou igual a $0,4 \times 0,4$, com assimetria menor que 0,2 entre os dois olhos. Quando um dos olhos de um mesmo paciente tinha sua escavação considerada como suspeita de glaucoma, automaticamente o olho contra-lateral também era excluído do estudo.

Todos os pacientes foram avaliados pelo mesmo examinador, que na seqüência submetia cada olho do paciente à análise da camada de fibras nervosas utilizando o analisador de fibras nervosas GDx (Laser Diagnostic Technology). Não foi realizado exame de perimetria computadorizada dada a baixa freqüência de defeitos de campo visual em pacientes portadores dos critérios de inclusão acima citados.

Eram obtidas 3 imagens de cada olho aceitas pelos critérios de aquisição do aparelho, e somente considerada para estudo a imagem média destas. Apenas as imagens médias com desvio-padrão menor que 8 micras eram aceitas para estudo.

Somente um dos olhos de cada paciente foi escolhido aleatoriamente para a análise dos dados, e a imagem média deste analisada utilizando-se os índices fornecidos pelo GDx, assim como os gráficos numérico e de cores.

As causas de exclusão foram impossibilidade em se obter três imagens de qualidade do olho em questão (qualidade esta avaliada pelo software do aparelho), um desvio-padrão maior que $8 \mu \mathrm{m}$ após a obtenção da imagem média, e imagens des- centradas, que poderiam dificultar a realização das medidas pelo aparelho.

De um total de 94 olhos (50 pacientes) foram excluídos 60 pelos critérios adotados acima, restando 34 olhos, que se constituíram na amostra deste estudo.

Definimos como normal aquele exame em que todos os índices já normatizados (o number não é normatizado) mostravam-se dentro de $90 \%$ da faixa de normalidade (incluindo-se os limítrofes ou "borderline"), e exame alterado aquele no qual pelo menos um dos 11 índices (não se considerando o number) se mostrava fora dos padrões de normalidade.

\section{RESULTADOS}

Obtivemos 20 exames normais e 14 exames alterados (denominados falsos-positivos), o que nos dá uma freqüência de falsos-positivos de $41,17 \%$, e uma especificidade de $58,82 \%$ (tabelas 1 e 2). A faixa etária dos pacientes com exames falsospositivos variou de 21 a 71 anos, com média de 41,73 anos, enquanto a dos pacientes com exame normal variou de 18 a 64 anos, com média de 34,72 anos.

Dentre os exames considerados falsos-positivos, o índice que se mostrou alterado com maior freqüência foi a simetria $(85,71 \%$ dos exames alterados), seguida pelo number $>30$ (50\%), superior average (42,85\%), superior integral $(35,71 \%)$, superior/nasal (35,71\%), elipse average (14,28\%) e average thickness $(7,14 \%)$. Os índices inferior ratio e superior ratio não apresentaram alteração em nenhum dos casos, e o inferior average e max. modulation em apenas um dos casos $(7,14 \%)$ (tabela 3).

O number variou, entre os exames alterados de 10 a 55 (média de 31,85), e, entre os exames normais de 07 a 37 (média de 16,15) (tabelas 1 e 2).

Dos 14 exames alterados, 05 mostraram redução significativa da camada de fibras nervosas $(\mathrm{p}<10 \%)$ apenas superiormente, enquanto 01 apresentou redução superior e nasal e 01 superior e temporal.

\begin{tabular}{|c|c|c|c|c|c|c|c|c|c|c|c|c|}
\hline Caso & Simetria & Sup.r. & Inf.r. & Sup/n & Max.m & EM & Number & AT & EA & SA & IA & SI \\
\hline $02 \mathrm{OE}$ & ALT. & NL & NL & NL & NL & $\mathrm{NL}$ & 10 & NL & NL & BORD & NL & ALT. \\
\hline 07OE & ALT. & NL & NL & ALT. & NL & NL & 50 & BORD & ALT & ALT & ALT & ALT. \\
\hline $08 \mathrm{OE}$ & ALT. & NL & NL & $\mathrm{NL}$ & NL & NL & 11 & BORD & NL & ALT & NL & ALT. \\
\hline 09OE & ALT. & NL & NL & NL & NL & NL & 11 & NL & NL & BORD & NL & BORD \\
\hline $19 O D$ & ALT. & BORD & NL & ALT. & NL & $\mathrm{NL}$ & 55 & BORD & BORD & $\mathrm{ALT}$ & NL & BORD \\
\hline $210 E$ & ALT. & NL & NL & ALT. & NL & $\mathrm{NL}$ & 53 & ALT. & ALT & ALT & BORD & ALT. \\
\hline 22OD & BORD & NL & NL & $\mathrm{NL}$ & NL & $\mathrm{NL}$ & 38 & BORD & BORD & ALT & NL & ALT. \\
\hline $290 E$ & ALT. & NL & NL & BORD & NL & $\mathrm{NL}$ & 15 & $\mathrm{NL}$ & NL & NL & NL & $\mathrm{NL}$ \\
\hline 40OD & ALT. & NL & NL & $\mathrm{NL}$ & NL & $\mathrm{NL}$ & 26 & NL & NL & $A L T$ & NL & BORD \\
\hline $44 O E$ & ALT. & NL & NL & BORD & NL & $\mathrm{NL}$ & 19 & NL & NL & BORD & NL & BORD \\
\hline $45 \mathrm{OE}$ & ALT. & BORD & NL & $\mathrm{NL}$ & BORD & $\mathrm{NL}$ & 55 & NL & NL & BORD & NL & $\mathrm{NL}$ \\
\hline $47 \mathrm{OE}$ & ALT. & BORD & NL & NL & NL & $\mathrm{NL}$ & 20 & NL & NL & $\mathrm{NL}$ & NL & $\mathrm{NL}$ \\
\hline $48 O D$ & ALT. & $\mathrm{NL}$ & NL & ALT. & NL & $\mathrm{NL}$ & 48 & NL & NL & NL & NL & $\mathrm{NL}$ \\
\hline $49 O D$ & BORD & BORD & NL & ALT. & ALT. & ALT & 35 & NL & NL & NL & NL & $\mathrm{NL}$ \\
\hline \multicolumn{13}{|c|}{$\begin{array}{l}\text { Alterados/total }=14 / 34=41,17 \% \text {; Especificidade total=20/34=58,82\%; Especificidade corrigida }=24 / 34=70,58 \% \text {; } N^{\circ} \text { alterados (Excetuando-se os casos sem redução } \\
\text { de fibras): } 10 \text { olhos; } N^{\circ} \text { alterados total: } 14 \text { olhos. } \\
\text { Legendas: Sup.r.- superior ratio ; Inf.r.- inferior ratio; Sup/n- superior/nasal; Max.m-max. modulation; EM- elipse modulation; AT- average thickness; EA- elipse } \\
\text { average; SA-superior average; IA- inferior average; SI- superior integral; NL-Normal; BORD- Borderline; ALT-alterado. }\end{array}$} \\
\hline
\end{tabular}




\begin{tabular}{|c|c|c|c|c|c|c|c|c|c|c|c|c|}
\hline \multirow[b]{2}{*}{ Caso } & \multirow[b]{2}{*}{ Simetria } & \multirow[b]{2}{*}{ Sup.r } & \multirow[b]{2}{*}{ Inf.r } & \multirow[b]{2}{*}{ Sup/n } & \multicolumn{8}{|c|}{ Tabela 2. Exames Normais } \\
\hline & & & & & Max.m & EM & Number & AT & EA & SA & IA & SI \\
\hline 06OD & BORD & $\mathrm{NL}$ & $\mathrm{NL}$ & BORD & $\mathrm{NL}$ & $\mathrm{NL}$ & 13 & NL & $\mathrm{NL}$ & NL & NL & NL \\
\hline 05OD & NL & NL & $\mathrm{NL}$ & BORD & BORD & BORD & 21 & NL & $\mathrm{NL}$ & $\mathrm{NL}$ & $\mathrm{NL}$ & NL \\
\hline $170 \mathrm{E}$ & $\mathrm{NL}$ & NL & NL & $\mathrm{NL}$ & $\mathrm{NL}$ & $\mathrm{NL}$ & 09 & $\mathrm{NL}$ & $\mathrm{NL}$ & NL & NL & NL \\
\hline $180 \mathrm{D}$ & $\mathrm{NL}$ & $\mathrm{NL}$ & $\mathrm{NL}$ & $\mathrm{NL}$ & NL & NL & 11 & NL & $\mathrm{NL}$ & $\mathrm{NL}$ & $\mathrm{NL}$ & BORD \\
\hline 20OD & BORD & $\mathrm{NL}$ & $\mathrm{NL}$ & $\mathrm{NL}$ & NL & NL & 19 & $\mathrm{NL}$ & $\mathrm{NL}$ & $\mathrm{NL}$ & $\mathrm{NL}$ & $\mathrm{NL}$ \\
\hline $230 D$ & $\mathrm{NL}$ & NL & $\mathrm{NL}$ & NL & NL & NL & 14 & NL & $\mathrm{NL}$ & $\mathrm{NL}$ & $\mathrm{NL}$ & NL \\
\hline $25 \mathrm{OD}$ & NL & NL & $\mathrm{NL}$ & $\mathrm{NL}$ & NL & NL & 09 & NL & $\mathrm{NL}$ & $\mathrm{NL}$ & $\mathrm{NL}$ & $\mathrm{NL}$ \\
\hline $270 E$ & BORD & $\mathrm{NL}$ & NL & NL & NL & NL & 14 & BORD & $\mathrm{NL}$ & NL & NL & BORD \\
\hline $300 D$ & $\mathrm{NL}$ & $\mathrm{NL}$ & $\mathrm{NL}$ & $\mathrm{NL}$ & $\mathrm{NL}$ & $\mathrm{NL}$ & 24 & $\mathrm{NL}$ & $\mathrm{NL}$ & NL & $\mathrm{NL}$ & BORD \\
\hline $310 E$ & BORD & NL & $\mathrm{NL}$ & NL & NL & NL & 37 & NL & $\mathrm{NL}$ & $\mathrm{NL}$ & $\mathrm{NL}$ & NL \\
\hline $32 \mathrm{OE}$ & $\mathrm{NL}$ & NL & $\mathrm{NL}$ & $\mathrm{NL}$ & $\mathrm{NL}$ & $\mathrm{NL}$ & 13 & NL & $\mathrm{NL}$ & $\mathrm{NL}$ & $\mathrm{NL}$ & NL \\
\hline $340 D$ & BORD & NL & $\mathrm{NL}$ & $\mathrm{NL}$ & $\mathrm{NL}$ & $\mathrm{NL}$ & 13 & NL & $\mathrm{NL}$ & NL & NL & $\mathrm{NL}$ \\
\hline $350 D$ & BORD & NL & NL & $\mathrm{NL}$ & $\mathrm{NL}$ & $\mathrm{NL}$ & 15 & NL & $\mathrm{NL}$ & NL & NL & NL \\
\hline $370 \mathrm{D}$ & NL & NL & $\mathrm{NL}$ & NL & $\mathrm{NL}$ & NL & 32 & NL & $\mathrm{NL}$ & $\mathrm{NL}$ & $\mathrm{NL}$ & NL \\
\hline $380 E$ & $\mathrm{NL}$ & BORD & $\mathrm{NL}$ & $\mathrm{NL}$ & BORD & NL & 21 & NL & $\mathrm{NL}$ & $\mathrm{NL}$ & $\mathrm{NL}$ & NL \\
\hline $390 E$ & BORD & NL & $\mathrm{NL}$ & $\mathrm{NL}$ & $\mathrm{NL}$ & $\mathrm{NL}$ & 11 & NL & $\mathrm{NL}$ & $\mathrm{NL}$ & $\mathrm{NL}$ & $\mathrm{NL}$ \\
\hline $410 \mathrm{D}$ & $\mathrm{NL}$ & $\mathrm{NL}$ & $\mathrm{NL}$ & $\mathrm{NL}$ & $\mathrm{NL}$ & $\mathrm{NL}$ & 07 & $\mathrm{NL}$ & $\mathrm{NL}$ & $\mathrm{NL}$ & $\mathrm{NL}$ & $\mathrm{NL}$ \\
\hline $42 \mathrm{OE}$ & $\mathrm{NL}$ & $\mathrm{NL}$ & NL & $\mathrm{NL}$ & $\mathrm{NL}$ & $\mathrm{NL}$ & 15 & $\mathrm{NL}$ & $\mathrm{NL}$ & $\mathrm{NL}$ & $\mathrm{NL}$ & NL \\
\hline $430 D$ & $\mathrm{NL}$ & NL & $\mathrm{NL}$ & $\mathrm{NL}$ & $\mathrm{NL}$ & $\mathrm{NL}$ & 18 & NL & $\mathrm{NL}$ & $\mathrm{NL}$ & $\mathrm{NL}$ & $\mathrm{NL}$ \\
\hline & & & & & & & & & & & & \\
\hline \multicolumn{13}{|c|}{ Legendas: Sup.r.- superior ratio ; Inf.r.- inferior ratio; Sup/n- superior/nasal; Max.m-max.modulation; EM- elipse modulation; AT- average thickness; EA- elipse } \\
\hline
\end{tabular}

\begin{tabular}{|lcc|}
\hline \multicolumn{3}{|c|}{$\begin{array}{c}\text { Tabela 3. Freqüência de alteração dos índices dentre os } \\
\text { exames falsos-positivos (N=14 olhos) }\end{array}$} \\
\hline \multicolumn{1}{|l|}{ Índice } & Número de Alterados & Freqüência \\
Simetry & 12 & $85,71 \%$ \\
Number>30 & 07 & $50,00 \%$ \\
Superior Average & 06 & $42,85 \%$ \\
Superior Integral & 05 & $35,71 \%$ \\
Superior/Nasal & 05 & $35,71 \%$ \\
Elipse Average & 02 & $14,28 \%$ \\
Average Thickness & 01 & $07,14 \%$ \\
Max. Modulation & 01 & $07,14 \%$ \\
Inferior Average & 01 & $07,14 \%$ \\
Elipse Modulation & 01 & $07,14 \%$ \\
Superior Ratio & 00 & $00,00 \%$ \\
Inferior Ratio & 00 & $00,00 \%$ \\
\hline
\end{tabular}

\section{DISCUSSÃO}

$\mathrm{O}$ analisador de fibras nervosas tem mostrado uma boa reprodutibilidade intra e inter-examinadores ${ }^{1,7}$.

Porém, como toda nova tecnologia incorporada ao nosso arsenal diagnóstico, requer a realização de estudos que venham determinar a confiabilidade e a importância dos seus resultados.

Em nosso estudo, a especificidade do analisador de fibras nervosas para os pacientes normais (não-glaucomatosos) foi de aproximadamente $58,82 \%$, enquanto Weinreb ${ }^{8}$ determinou uma especificidade de $57 \%$.

Neste mesmo estudo, Weinreb mostra ainda que a simetria é o índice que menos nos permite separar um grupo de pacientes normais de outro com defeitos iniciais ou moderados de glaucoma. Este dado é corroborado pelo nosso achado de que dentre os exames falsamente positivos, a simetria foi o índice que se mostrou alterado com maior freqüência $(85,71 \%)$. Portanto, frente a um paciente com pressões intra-oculares normais e um nervo óptico sem sinais característicos de glaucoma, mas com um GDx onde apenas a simetria se mostra alterada, devemos levantar a possibilidade de um resultado falsamente positivo.

Dentre os 5 casos que tiveram como único índice alterado a simetria, 3 não mostraram perda localizada de fibras na análise do diagrama de probabilidades $(\mathrm{p}<10 \%)$, mas sim grande diferença entre a espessura das fibras das regiões superior e inferior. Esta diferença, nestes casos, deveu-se a um excesso localizado de fibras inferiormente.

Em outro olho do nosso estudo, o excesso difuso de fibras detectado no diagrama de probabilidades e no perfil da camada de fibras nervosas foi suficiente para que os índices simetria e superior/nasal (caso 48 OD) mostrassem valores com $\mathrm{p}<5 \%$. Este caso ilustra a importância de se fazer uma análise conjunta dos índices e diagramas fornecidos, sem a qual poderíamos inferir num falso diagnóstico.

Se no cálculo da especificidade (razão do número de exames normais pelo número total de exames) considerarmos como normais os 4 exames falsos-positivos que assim se apresentaram devido ao excesso de fibras nervosas ( 3 deles apenas inferiormente e 1 deles difusamente), teríamos um aumento da especificidade (agora chamada corrigida) de $58,82 \%(20 / 34)$ para $70,58 \%(24 / 34)$.

Em outro estudo (comunicação pessoal Remo Susanna Jr, M.D., 1999), onde foram submetidos à analise da CFN 40 pacientes com diagnóstico confirmado de glaucoma primário de ângulo aberto (através de campo visual automatizado e estereofotos), a simetria mostrou-se alterada em apenas $10 \%$ dos olhos. 
Os índices que mostraram menor freqüência de alteração foram o inferior ratio (0\%), superior ratio (0\%), elipse modulation $(7,14 \%)$, inferior average $(7,14 \%)$, max. modulation $(7,14 \%)$, average thickness $(7,14 \%)$ e elipse average $(14,28 \%)$. Estudos mostram que o melhor índice isolado para se suspeitar de um caso inicial de glaucoma é o inferior ratio, apesar dele somente fazer esta suspeita em $25 \%$ dos casos ${ }^{7}$. Na prática, devemos lembrar da hipótese de glaucoma sempre que um indivíduo suspeito tenha uma alteração do inferior ratio, já que este mostrou uma boa especificidade na classificação de um paciente como normal em nosso estudo.

Em um trabalho realizado em $1998^{9}$ encontraram o number como sendo o melhor índice para o diagnóstico de glaucoma.

Por fim, parece ser a menor espessura da camada de fibras nervosas da retina na área superior a maior responsável pelos casos de exames alterados entre os pacientes normais.

\section{CONCLUSÕES}

Os autores concluem que neste estudo, a especificidade do analisador de fibras nervosas para pacientes não-glaucomatosos foi de aproximadamente $59 \%$.

A simetria foi o índice que apresentou maior freqüência de alteração nos pacientes normais, sendo responsável por boa parte dos resultados falsos-positivos. Assim é que, frente a um paciente sem sinais oftalmoscópicos ou campimétricos de glaucoma, e com uma alteração da simetria isoladamente à análise da camada de fibras nervosas, devemos levantar a hipótese de um exame falso-positivo. Por sua vez, o inferior ratio raramente se mostra alterado em pacientes normais, e sua alteração associada a um disco óptico suspeito de glaucoma, é um fator a ser valorizado.

Finalmente, concluímos que o analisador de fibras nervosas GDx carece de outros estudos com maior amostragem que mostrem sua real importância em meio ao nosso arsenal no diagnóstico de glaucoma.

\section{ABSTRACT}

Purpose: To determine the fiber layer analyzer GDx's specificity in a group of normal subjects, and the most altered parameters for this group. Methods: Normal patients were examined (complete ophthalmologic examination) and then we performed the fiber layer analysis with NFA-GDx (LDT). Total: 34 randomly selected eyes. Results and Conclusion: The specificity was $58.8 \%$, and the corrected specificity $70.5 \%$. The most altered parameter was symmetry $(85.71 \%)$, and the less altered, the inferior and superior ratio (0\%). When we have an isolated altered symmetry in a patient with normal IOP and normal optic disc, the false-positive diagnosis must be remembered; when a suspected glaucoma patient has an altered inferior ratio, glaucoma diagnosis must be remembered.

Keywords: Nerve fibers; Sensitivity and specificity; Diagnostic techniques ophthalmological; Laser/diagnostic use.

\section{REFERÊNCIAS}

1. Weinreb RN, Shakiba S, Zangwill L. Scanning laser polarimetry to measure the nerve fiber layer of normal and glaucomatous eyes. Am J Ophthalmol 1995; 119:627-36.

2. Weinreb RN, Dreher AW, Coleman A, Quigley H, Shaw B, Reiter K. Histopathologic validation of Fourier-ellipsometry measurements of retinal nerve fiber layer thickness. Arch Ophthalmol 1990;108:557-60.

3. Dreher AW, Reiter K. Scanning laser polarimetry of the retina nerve fiber layer. SPIE Proc 1992;1746:34-8.

4. Weinreb RN, Shakiba S, Sample PA, Shahrokni S, van Horn S, Garden VS et al. Association between quantitative nerve fiber layer measurement and visual field loss in glaucoma. Am J Ophthalmol 1995;120:732-8.

5. Chi Q, Tomita G, Inazumi K, Hayakawa T, Tadayoshi I, Kitazawa Y. Evaluation of the effect of aging on the retinal nerve fiber layer thickness using scanning laser polarimetry. J Glaucoma 1995;4:406-13.

6. Tjon-Fo-Sang MJ, de Vries J, Lemij HG. Measurement by nerve fiber analyser of retinal nerve fiber layer thickness in normal subjects and patients with ocular hypertension. Am J Ophthalmol 1996;122:220-7.

7. Duarte FB, Paranhos AJR, Galhardo RA, Abdanur LRA, Prata JA, Mello PAA. Reprodutibilidade do exame de medida da camada de fibras nervosas da retina por meio da polarimetria de varredura a laser. Arq Bras Oftalmol 1999; 62:555-60.

8. Weinreb RN, Zangwill L, Berry CC, Bathija R, Sample PA. Detection of glaucoma with scanning laser polarimetry. Arch Ophthalmol 1998;116:1583-9.

9. Susanna Jr R, Takahashi WY, Nakamura NKF. Sensibilidade e especificidade da avaliação da camada de fibras nervosas examinadas através da polarimetria a laser. Rev Bras Oftalmol 1998;57:17-22.

\section{Encontro da ARVO}
Association for Research in Vision and Ophthalmology 29 de Abril a 4 de Maio de 2.001
Fort Lauderdale - Flórida - EUA
INFORMAÇÕES: home page : www.arvo.org/arvo 